\title{
Workload Prediction for Adaptive Power Scaling Using Deep Learning
}

\section{Citation}

Tarsa, Stephen J., Amit Kumar, and H.T. Kung. 2014. Workload Prediction for Adaptive Power Scaling Using Deep Learning. In 2014 IEEE International Conference on Integrated Circuit Design and Technology (Austin, TX, May 28-30, 2014), 1-5. Piscataway, NJ: IEEE.

\section{Published Version}

doi:10.1109/ICICDT.2014.6838580

\section{Permanent link}

http://nrs.harvard.edu/urn-3:HUL.InstRepos:12415311

\section{Terms of Use}

This article was downloaded from Harvard University's DASH repository, and is made available under the terms and conditions applicable to Open Access Policy Articles, as set forth at http:// nrs.harvard.edu/urn-3:HUL.InstRepos:dash.current.terms-of-use\#OAP

\section{Share Your Story}

The Harvard community has made this article openly available.

Please share how this access benefits you. Submit a story.

\section{Accessibility}




\title{
Workload Prediction for Adaptive Power Scaling Using Deep Learning
}

\author{
Stephen J. Tarsa, Amit P. Kumar, and H.T. Kung \\ Harvard University, Cambridge, MA \\ Intel Corporation, Microarchitectures Research Lab, Santa Clara, CA
}

\begin{abstract}
We apply hierarchical sparse coding, a form of deep learning, to model user-driven workloads based on on-chip hardware performance counters. We then predict periods of low instruction throughput, during which frequency and voltage can be scaled to reclaim power. Using a multi-layer coding structure, our method progressively codes counter values in terms of a few prominent features learned from data, and passes them to a Support Vector Machine (SVM) classifier where they act as signatures for predicting future workload states. We show that prediction accuracy and look-ahead range improve significantly over linear regression modeling, giving more time to adjust power management settings. Our method relies on learning and feature extraction algorithms that can discover and exploit hidden statistical invariances specific to workloads. We argue that, in addition to achieving superior prediction performance, our method is fast enough for practical use. To our knowledge, we are the first to use deep learning at the instruction level for workload prediction and on-chip power adaptation.
\end{abstract}

\section{INTRODUCTION}

Mechanisms like dynamic voltage and frequency scaling (DVFS) enable adaptive power management, and promise to improve operating efficiency by tailoring device parameters to workloads at runtime. Such adaptation requires anticipating future circuit states, and online workload modeling is one predictive approach that minimizes static or steady-state assumptions about workloads. This flexibility is important since performance characteristics like power consumption vary widely by user and application mix [1] [2].

In this paper, we use statistical relationships learned from hardware performance counters to predict periods of low instruction throughput, during which voltage and frequency can be scaled to reclaim power. We target predictions that are long-range and low-latency, meaning that look-ahead time is maximized to allow for chip adjustment, and predictive models update quickly when workloads change.

The most popular method for workload prediction is regression [3] [4] [5], which fits a polynomial to counter measurements and extrapolates future states. However, we show that regression accuracy degrades at long ranges, as future states are unlikely to be a simple extrapolation of prior measurements. Moreover, to capture fine-grained behaviors, regression coefficients must continually be updated even if the high-level workload is the same.

Instead, we implement modeling and prediction using hierarchical sparse coding and Support Vector Machine (SVM) classification, as depicted in Figure 1. This approach first codes counter measurements in terms of a few atoms selected from a

This material is based on research sponsored in part by the Intel Corporation (C) 2014 IEEE - ICICDT'14 dictionary of patterns, or features, learned from training data. These feature vectors are then concatenated and coded again to capture feature interrelationships over a larger spatial scale. An SVM classifies the resulting sparse vectors with a common label when they precede an event of interest.

The process of sparse coding cuts away noise from measurement data, and improves SVM classification accuracy when data is subject to non-Gaussian variations. In addition, hierarchical models can capture statistical patterns embedded in large state spaces from a modest number of training examples. We show that training time can be reduced even more by bootstrapping feature dictionaries using a canonical set of Layer 1 features, which are common across workloads. As a result, when workloads change, only a small number of training samples are required to update our predictor.

We adopt hierarchical sparse coding to capture complicated signature patterns appearing over time, and show that this improves prediction range over regression and heuristic techniques. This is because data vectors that have been hierarchically sparse coded are classified in a transformed domain: the feature space. By performing statistical inference on feature vectors, we exploit workload-specific invariant patterns that are typically "hidden," or not immediately observable in the raw data domain. In this paper, we use clustering on training data to extract this hidden structure. This form of deep learning has yielded major application gains in fields like computer vision, speech recognition, and machine translation (e.g. [6] [7]). To our knowledge, we are the first to apply sparse hierarchical models to chip performance data for adaptive optimization.

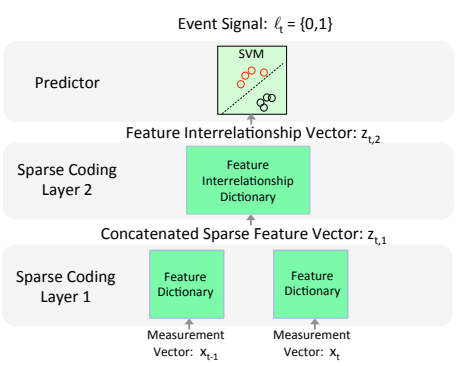

Fig. 1. We sparse-code vectors of counter values in two layers to extract patterns over time, and use SVM classification to identify events of interest.

\section{TARGET SCEnARIO AND DATA COLlECTION}

\section{A. Device, Workloads, and Event Prediction}

We collect performance counter data using gem5, an architecture-level simulator with full system support, including frame buffer rendering and an interactive shell [8]. Snapshots of counter values are taken every $500 \mu$ s from a simulated 1.0 $\mathrm{GHz}$ ARM v7a chip. 


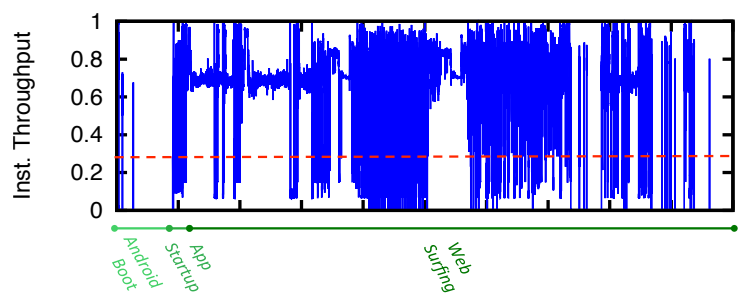

Fig. 2. A plot of committed instructions per cycle for BBENCH shows that nearly $20 \%$ of the web surfing phase exhibits low instruction-throughput, and is a target for DVFS. These intervals arise due to data $\mathrm{I} / \mathrm{O}$, and also intermittently during computations, due to architecture-level interactions of chip components. We use deep learning to find predictive signatures for this latter class of dips, which make up $7.3 \%$ of the surfing phase.

Our primary workload is the BBENCH benchmark [9], running atop Android Gingerbread. Figure 2 plots committed instructions/cycle for three phases of activity: OS boot, web browser startup, and a web surfing phase. During surfing, web sites are loaded from off-chip using Android's built-in browser, and Javascript code simulates user link clicks. We see that instruction throughput drops below $25 \%$ during nearly $20 \%$ of the surfing phase, making these intervals a good target for DVFS. Here, low instruction throughput arises under two sets of circumstances: first, while waiting on web page I/O, and second, during computation-dominated intervals due to the architecture-level interactions of chip components. This latter class of intermittent dips make up $7.3 \%$ of the overall workload, and are difficult to predict with heuristics. By applying hierarchical sparse coding, we will find predictive signatures for these dips, and reclaim power by voltage/frequency scaling.

To generalize gains from our approach, we also report prediction performance for the ASIMBENCH/Moby benchmark suite [10]. This set of workloads includes examples of a game, audio and video playback, and document manipulation applications running under Android ICS. Though these workloads capture additional application behaviors, they lack the simulated user interactions of BBENCH that cause pertinent statistical patterns to recur over time. We therefore present these limited results with the caveat that incorporating user interaction is a necessary next-step to assess power savings.

Our workload prediction scenario is related to the wellstudied problem of workload phase detection. Phase detection is often motivated by the desire to identify large stable regions of a workload, which ensure that overheads resulting from optimization-driven adjustments can be sufficiently amortized. Detection techniques like working set signatures, basic block vectors, and conditional branch counters (see [11] for a review) apply a threshold to one or more hardware counters to identify deviations relative to a long-term average. We will show that more sophisticated statistical techniques are necessary to implement long-range prediction.

However, phase detection can serve as a useful complimentary technique. This lightweight method for detecting changes in long-term workload characteristics can trigger additional training to update our statistical models. Futhermore, as we will show in Section IV B, short unstable workload regions have few prediction opportunities, since the number of recurrent patterns is limited; detecting rapid phase changes is one potential way of short-circuiting ineffective predictions within these regions.

\section{B. Performance Counter Configuration}

Our sparse-coding predictor will use deep learning to discover signatures that span multiple measurement windows over time, and multiple counters across the chip. Counters capture events like committed instructions, data table hits, misses, flushes, etc. This approach contrasts with standard regression modeling that focuses on one or two counters most correlated with the metric of interest, e.g. in [4].

Typically, the choice of performance counters to include on a chip is based on both the desired performance monitoring data, as well as layout and design constraints. In this section, we use gem5 to also characterize the statistical properties of counter configurations. This allows us to choose a small number of counters that still give good prediction performance.

First, we collect data from 120 simulated hardware counters during the execution of our test workloads. This data represents a superset of possible hardware configurations. Every $500 \mu \mathrm{s}$, deltas from previous values are recorded, and we use the resulting data vectors to calculate a correlation matrix. We then group together counters whose correlational magnitude exceeds 0.98 , since these counters are statistically interchangeable due to their near-total correlation. By choosing one counter from each group, we then have a minimal configuration that provides comprehensive architecture-level statistics.

We identify 34 different groups from the 120 possible counters studied on our ARM v7a-chip. We find, for example, that the number of integer register reads is interchangeable with the number of committed integer operations, though these are collected from different locations on the chip. Such statisticsdriven counter selection is useful to control the data-collection overhead of our predictor without sacrificing accuracy.

Principal Component Analysis (PCA), a standard technique for dimensionality reduction, was also applied. Even though PCA found a lower dimensional basis for our data, that representation relies on linear combinations of all 34 counters. Therefore, this analysis does not allow us to reduce the number of counters deployed on-chip, though it implies that compressive techniques such as random linear combination are worth investigating to reduce acquisition costs [12] [13].

\section{HIERARCHICAL SPARSE CODING}

Sparse coding [14] formalizes dictionary learning and feature extraction by the following minimization:

$$
\min \|X-D Z\|_{2} \quad \text { s.t. }\left\|z_{i}\right\|_{0} \leq k \text { for } i=1 \ldots t
$$

with $X$ an $n \times t$ data matrix containing $t$ snapshots of $n$ counters, $D$ an $n \times m$ dictionary of $m$ features, $Z$ a sparse $m \times t$ matrix of feature coefficients, and $k$ a sparsity constraint. We also put non-negativity constraints on $D$ and $Z$ to improve coding stability for classification under data variation [7].

Sparse coding generalizes $k$-means, a method for finding clusters in data and representing vectors by their associated cluster centroid [15]. In sparse coding, when $k=1$, cluster centroids become dictionary atoms, and data-to-cluster assignments correspond to $Z$ 's coefficients. When $k>1$, a data vector is represented by a sparse, positive, linear combination of $k$ cluster centroids, rather than just one. K-SVD [14] trains $D$ by iteratively fixing $Z$ and using rank-one approximation to update $D$ 's columns, and then fixing $D$ to update $Z$ by 
Orthogonal Matching Pursuit (OMP) [16]. After training, OMP computes sparse representations relative to $D$ for new data vectors.

We choose sparse coding over alternative learning methods such as Convolutional Neural Networks for several reasons: first, a sum of commonly occurring patterns is an appropriate intuitive model for performance counters on a chip with multiple concurrently operating functional circuits. Second, sparse coding yields good classification performance using a linear SVM when few labeled training examples are available [7]. And third, both K-SVD and OMP consist primarily of innerproduct computations that can be built in hardware, e.g. [17].

We sparse code data hierarchically, as depicted in Figure 1. At Layer 1, canonical features present in $500 \mu s$ measurement windows are extracted from raw counter data. At Layer 2, we concatenate sparse feature vectors from multiple measurement windows over time, and again cluster vectors to capture feature co-occurrences. Finally, the outputs of Layer 2 are fed to a linear SVM that implements prediction by assigning a common label to vectors preceding our target event. When detecting one among many events, multiple SVMs or decision trees can be used at this last step. As previously mentioned, we convert each snapshot of counter values into a delta from the previous measurement window. Furthermore, we normalize values to lie between 0 and 1 , ensuring that learning using distance minimization treats approximation error in all counters equally.

Sparse hierarchical models are a primary driver of breakthroughs associated with deep learning for three major reasons. First, imposing sparsity on signals is a powerful denoising step that is critical when dealing with natural data variations. Second, by hierarchically learning features and their interrelationships, the model can express feature combinations not directly represented in training data. This means that a few training examples can be generalized for good statistical performance on a larger data set. Third, hierarchical models often include a non-linear pooling step that corrects for alignment variations by maximizing feature response over shifted measurement patches, similar to a convolutional filter. In this paper, we found little gain from pooling since gem5's timing is deterministic and repeatable, however we expect this tool be important when we expand to data measured from hardware.

\section{WORKLOAD PREDICTION}

\section{A. BBENCH Performance}

We first present results comparing prediction performance between hierarchical sparse coding, linear regression, and static heuristics, for detecting sub-25\% instruction-throughput dips during BBENCH. We define prediction accuracy as the portion of all $500 \mu s$ windows that are correctly labeled, based on whether their instruction throughput is above or below 25\%:

$$
\text { Accuracy }=\frac{\text { True Positives }+ \text { True Negatives }}{\text { Total \# of Windows Classified }}
$$

We also care about false alarm statistics, especially since there is a recovery cost associated with false positive alarms. We therefore measure precision and recall. Precision is the number of correctly predicted dips over the total number of alarms:

$$
\text { Precision }=\frac{\text { True Positives }}{\text { True Positives }+ \text { False Positives }}
$$

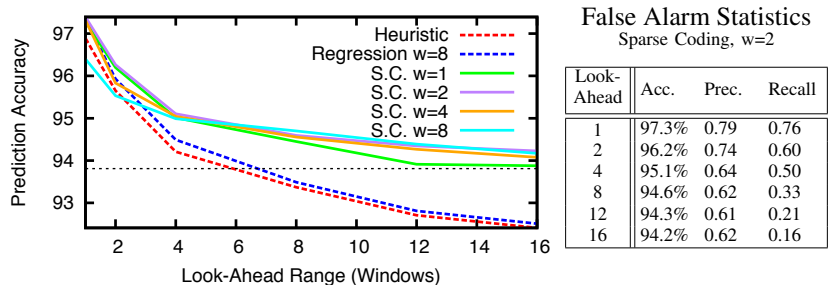

Fig. 3. Throughput dips make up nearly $7.3 \%$ of the surfing phase's computations, and the dotted black line depicts accuracy if we make no positive dip predictions at all. Curves above this line have positive predictive value overall, whereas curves below the dashed line make enough incorrect predictions to be worse than doing nothing. Precision and recall are shown for sparse coding over $\mathrm{w}=2$ windows, and characterize the value of positive dip predictions: precision tells how often positive alarms are correct, and recall tells how many dips that occur in the workload are correctly identified. For example, curves below the dashed do-nothing baseline have prec. $<0.50$, since positive alarms are mistaken on average.

Recall is the number of correctly predicted dips over the total number of dips that actually occur during the workload:

$$
\text { Recall }=\frac{\text { True Positives }}{\text { True Positives }+ \text { False Negatives }}
$$

Sparse coding dictionaries are trained using the entire web surfing phase of our dataset, and SVM accuracy is measured under cross-fold validation. This captures the best-case scenario, in which data volume is always sufficient for training, regardless of acquisition time. We code over $w=1,2,4,8$ trailing measurement windows of $500 \mu \mathrm{s}$ snapshots to find predictive effects over different time scales. Since parameters such as dictionary size and sparsity constraints may impact performance, we test a wide range of model configurations, and skim off the best performing (we analyze how model configuration affects performance in Section VI).

To compare, we fit regression coefficients for linear predictors of different orders based on the instruction throughput metric directly. In Figure 3, we show data from an order-8 regression, which performed best. Regressions are computed using a sliding window of measurement data, and curves are extrapolated and thresholded to implement dip prediction.

We also compare to a heuristic based on the observation that one dip often precedes another. The heuristic waits until a dip has been observed, and naïvely assumes that another will follow. This represents the simplest predictor.

Figure 3 plots overall prediction accuracy against lookahead range. First, we see that the static heuristic works only at short look-ahead ranges, when it can pick up the latter portions of dips spanning multiple measurement windows. Regression successfully extends prediction range, and captures dips that can be extrapolated from prior measurements. However, sparse coding consistently has best accuracy and range for lookahead ranges of 3 windows or more. Furthermore, we see that regression and heuristics make so many mistakes at long ranges that they are worse than doing nothing. In contrast, sparse coding always has positive predictive power, even at a range of 16 windows. Practically, by extending look-ahead range from 6 windows to 16 , range is increased by almost $3 x$.

\section{B. ASIMBENCH/Moby Performance}

In this section, we present prediction accuracy for workloads in the ASIMBENCH/Moby benchmark suite, which include a video game, audio and video playback, and viewers 
TABLE I. PREDICTION ACCURACY BY WORKLOAD

\begin{tabular}{|c|c|c|c|c|c|}
\hline \multirow{2}{*}{ Workload Name } & \multirow{2}{*}{ Phase } & \multirow{2}{*}{ Description } & \multirow{2}{*}{$\begin{array}{l}\text { \% Low Instr. } \\
\text { Throughput }\end{array}$} & \multicolumn{2}{|c|}{ S.C. Pred. Acc. } \\
\hline & & & & Look-Ahead $=2$ & Look-Ahead $=4$ \\
\hline Adobe Reader & All & $\begin{array}{l}\text { Display PDF file } \\
\end{array}$ & $11.2 \%$ & $92.2 \%$ & $89.9 \%$ \\
\hline \multirow{2}{*}{ Frozen Bubble } & Phase I & \multirow{2}{*}{ Initialize and begin game } & $13.6 \%$ & $92.0 \%$ & $88.9 \%$ \\
\hline & Phase 2 & & $64.6 \%$ & $94.0 \%$ & $86.0 \%$ \\
\hline k9 Mail & All & Display e-mails & $19.3 \%$ & $89.6 \%$ & $86.5 \%$ \\
\hline \multirow{3}{*}{ KingSoft Office } & Phase 1 & \multirow{3}{*}{ Open .doc/.xls/ppt files } & $14.9 \%$ & $88.7 \%$ & $85.5 \%$ \\
\hline & Phase 2 & & - & - & - \\
\hline & $\begin{array}{l}\text { Phase } 3 \\
\text { Phase } 4\end{array}$ & & $70.2 \%$ & $93.4 \%$ & $85.4 \%$ \\
\hline \multirow{3}{*}{ MXPlayer } & Phase I & \multirow{3}{*}{ Play a video } & $13.8 \%$ & $91.0 \%$ & $88.0 \%$ \\
\hline & Phase 2 & & - & - & - \\
\hline & Phase 3 & & $6.6 \%$ & $98.7 \%$ & $97.8 \%$ \\
\hline \multirow{2}{*}{ ttpod } & Phase I & \multirow[t]{2}{*}{ Play mp3 } & $13.0 \%$ & $91.1 \%$ & $88.7 \%$ \\
\hline & Phase 2 & & $11.8 \%$ & $97.6 \%$ & $95.1 \%$ \\
\hline
\end{tabular}

for PDF and Microsoft Office documents. For each workload, we report the percentage of computation time during which instruction throughput is below $25 \%$, and the prediction accuracy using signatures spanning 2 windows.

Applying a phase detection filter as discussed in Section II, we see that some workloads can naturally be divided into distinct stable regions. For example, the Frozen Bubble video game has two phases: in the first, application data is loaded and game state initialized, and in the second, the game enters a regular frame-rendering loop. We therefore break out performance per phase, and report only on workload regions with at least 8,000 measurement snapshots. This conservatively ensures that models can be trained. Benchmark descriptions and overall prediction accuracy are shown in Table I.

Hierarchical sparse coding performs best when workloads have recurring patterns. In this set of benchmarks, media workloads that are cyclic and driven by a regular sampling rate have best prediction performance. For Frozen Bubble Phase 2, MXPlayer Phase 3, and ttpod Phase 2, dip prediction is nearly perfect: $94.0 \%, 98.7 \%$, and $97.6 \%$, respectively.

For Frozen Bubble and ttpod, an order- 8 regression yields sub-60\% accuracy, indicating that cyclic dips are not extrapolations of prior counter values sampled every $500 \mu \mathrm{s}$. Though adapting measurement sampling rate to workloads may improve regression, our method needs no such workload-specific adjustment. Furthermore, adjustment may not be possible in cases like video streaming or games, since frame rates are variable, and affected by background computation and I/O.

We also find that short phases with a high degree of irregular variation lead to few useful long-range signatures. Fitting this description are the k9 Mail benchmark, and most applications' initialization Phase 1's. Here, phase detection for stable-region identification could be used to short-circuit predictions that are unlikely to be useful. However, for $\mathrm{k} 9$ Mail, we note that the lack of user interaction over a large time scale is a potential limit on prediction opportunities.

\section{Dynamic POWER FOR Voltage ScAling}

The false negative and false positive rates of a predictor impact power savings due to missed opportunity costs and recovery costs for incorrect scaling decisions. Given these, we model realized dynamic power during instruction throughput dips when our predictive frequency/voltage scaling is applied:

$$
\begin{aligned}
P_{\mathrm{dyn}} & =\operatorname{Pr}(\text { True Pos. }) *\left(V_{r d}\right)^{2}\left(f_{r d}\right)\left(a_{r d}\right) \\
& +\operatorname{Pr}(\text { False Neg. }) *\left(V_{o}\right)^{2}\left(f_{o}\right)\left(a_{r d}\right) \\
& +\operatorname{Pr}(\text { False Pos. }) *\left(V_{o}\right)^{2}\left(f_{o}\right)\left(a_{o}+a_{p l t}\right)
\end{aligned}
$$

For simplicity, this model assumes constant capacitance and linear frequency/voltage scaling. As in [18], we proportionally scale power by the amount of realized switching activity in

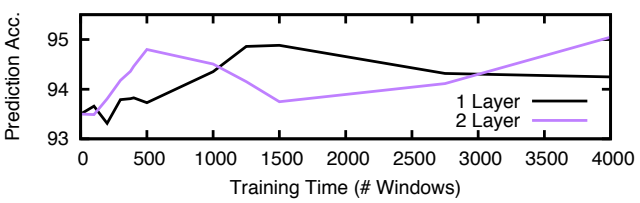

Fig. 5. Initially, sparse coding models initially converge to a local maximum prediction accuracy, based on the predictive power of single-window features. When more variations driven by underlying interrelationships are observed, additional training is needed to capture their predictive power. A 2 layer model learns these second-order effects from roughly $3 x$ fewer training samples.

different scenarios. $a_{o}=0.75$ represents ordinary operation, based on the observed average activity during BBENCH. $a_{r d}$ represents reduced activity during dips in instruction throughput. Finally $a_{p l t}$ represents additional penalty activity to compensate for incorrect scaling decisions.

When instruction throughput drops, gating is used to reduce switching activity. However, gating mechanisms are imperfect and design specific, so we use a gating efficiency term $g$ to compare different scenarios. $a_{r d}$ is therefore defined as $a_{r d}=$ $1.0-(0.75) * g$ for an instruction throughput drop of $75 \%$.

The model consists of terms representing power consumption for correct predictions, false negatives, and false positives, respectively. When a dip is correctly predicted, voltage and frequency are reduced from $V_{o}=1.0$ to $V_{r d}=0.25$, and $f_{o}=1.0$ to $f_{r d}=0.25$. For false negatives, voltage and frequency remain at $V_{o}=1.0$ and $f_{o}=1.0$. For false positives, we penalize incorrect scaling with increased switching activity: $\left(a_{o}+a_{p l t}\right)$. This assumes that we detect higher-than-predicted activity, and execute additional recovery steps.

Figure 4 plots $P_{\text {dyn }}$ per dip for our best sparse coding model, against look-ahead range. Gating efficiency establishes the do-nothing baseline power consumption during a dip, so we vary $g=0.15 \ldots 0.66$ to capture savings for a range of chip designs. Power savings are also parameterized by the recovery cost $a_{p l t}$, which we vary from $+10 \%$ to $+40 \%$ switching activity. When the recovery cost is $+25 \%$ activity, predictive voltage scaling successfully reduces power consumption with a 4 window heads up, or $2 \mathrm{~ms}$. If we can tolerate only a $1 \mathrm{~ms}$ chip adjustment time, then this savings is a $50 \%$ gain over a $g=0.33$ gating-efficient design without voltage scaling.

\section{DictionaRY TRAINING AND CONFIGURATION}

For low-latency prediction, we must ensure that sparse coding dictionaries can be trained quickly under changing workloads. To this end, we first demonstrate the impact of hierarchy on training time. Figure 5 shows prediction accuracy as the number of training samples increases, comparing a 2 layer hierarchical model to an equivalent 1 layer model that codes concatenated measurement vectors directly.

Prediction accuracy for both models follows the same basic shape. With few training samples, predictors capture singlewindow effects, and converge to a local maximum. As data is added, variations arising from underlying feature interrelationships are observed. Initially, this added data complexity dilutes the training set, hurting prediction. However, when enough samples are acquired to fully capture second-order effects, prediction converges to a new, higher maximum. Here, we see that hierarchically treating feature interrelationships reduces training data requirements by roughly $3 \mathrm{x}$. 

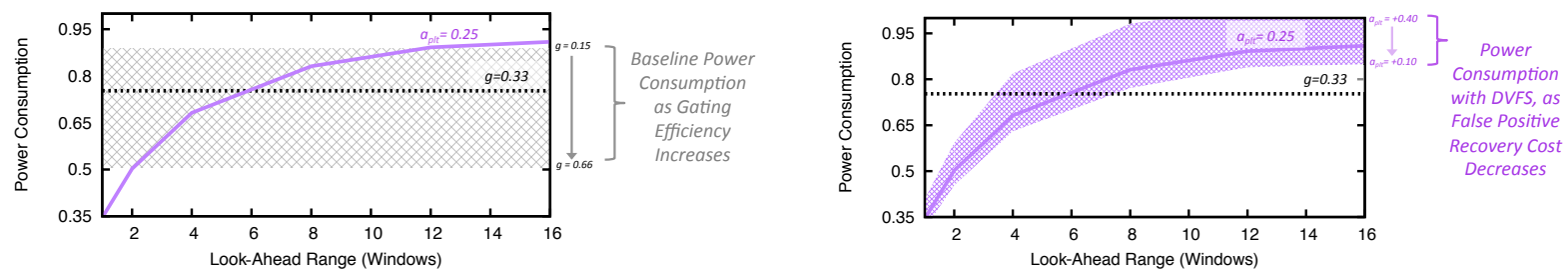

Fig. 4. Our power model measures savings during dips relative to a chip's baseline do-nothing power consumption. On the left, baseline power consumption is parameterized by a chip's gating efficiency $g$; when gating efficiency is low (e.g. $g=0.15$ ), then do-nothing power consumption is higher, and predictive DVFS leads to significant power reduction. On the right, power under predictive DVFS is partly determined by the cost of false positive recovery, $a_{p l t}$; if false positives can be corrected-for with a small amount of additional switching activity (e.g. $a_{p l t}=+0.25$ ), then the gain of predictive DVFS is high.

TABLE II. Prediction AcCuracy By Model CONFiguration

\begin{tabular}{|r||c|c||c|c|c|}
\hline Config. & $\begin{array}{c}\text { Layer 1 } \\
\text { \# Dict Atoms }\end{array}$ & $\begin{array}{c}\text { Layer 2 } \\
\text { \# Dict Atoms }\end{array}$ & Acc. & Prec. & Recall \\
\hline \hline Baseline & 100 & 100 & $94.4 \%$ & $58 \%$ & $38 \%$ \\
Small Layer 1 Dict. & 30 & - & $94.7 \%$ & $62 \%$ & $37 \%$ \\
Small Layer 2 Dict. & - & 30 & $94.2 \%$ & $56 \%$ & $30 \%$ \\
\hline
\end{tabular}

Small Layer 1 dictionaries improve precision, possibly from better de-noising. Large Layer 2 dictionaries are needed to capture feature interrelationships, shown by recall.

TABLE III. LAYER 1 TRAINING BY WORKLOAD MIX

\begin{tabular}{|l|l|c|}
\hline Layer 1 Training Workload & Layer 2 Training Workload & Pred Acc. \\
\hline \hline BBENCH & BBENCH & $94.5 \%$ \\
\hline Adobe Reader & BBENCH & $93.4 \%$ \\
King Soft (Phase 4) & BBENCH & $94.2 \%$ \\
Bootstrapped (Rand. Sample) + BBENCH & BBENCH & $94.7 \%$ \\
\hline
\end{tabular}

Training Layer 1 using evenly mixed workloads leads to best performance.

Training time is also driven by dictionary sizes, a choice with implications for overall prediction accuracy. Large dictionaries need more data to train more states. However, they can over-fit if extra atoms capture patterns that do not meaningfully reflect data clusters. In contrast, small dictionaries may only have capacity to reflect local workload regions; when trained over too large a region, atoms become coarse averages of many features, washing out nuanced predictive effects.

In Table II, we examine the effect of changing Layer 1 and Layer 2 dictionary sizes. When we fix the Layer 1 dictionary to be small, we see that accuracy, precision, and recall change little relative to a much larger Layer 1 dictionary. This suggests that a few canonical features are enough to characterize complicated workloads. In contrast, when we fix a small Layer 2 dictionary, recall drops relative to a much larger dictionary. This indicates that a larger Layer 2 dictionary is required to capture interrelationships among canonical features.

The utility of a small Layer 1 dictionary suggests that a few features are sufficient even for complicated workloads, so we examine if those features are universal. Table III shows prediction accuracy when the Layer 1 dictionary is trained on different workloads, with a 4 window look-ahead, and 2window signatures. We see that, even when the Layer 1 dictionary is trained on out-of-band samples, useful prediction is realized. Furthermore, bootstrapping on a mix of samples from all workloads improves performance over training directly on BBENCH. We conclude that a canonical set of Layer 1 features exists across workloads, and that they can be burned-in over a long period, and updated infrequently.

\section{CONCLUSION \& FUtURE WORK}

Deep learning holds much promise for workload modeling and adaptive chip optimization. We believe this study is an important first step to begin exploring those opportunities.
Three major directions for future work exist. First, we must apply this methodology to more workloads, with user interaction as a first-class characteristic. Second, we will experimentally gather data from a hardware platform to verify that predictive performance holds when timing and measurement errors seep into data. We expect non-linear pooling, mentioned in Section III, to be important in this context. Finally, predictive DVFS is a first-cut use case, and we will look at other scenarios to which we can apply deep learning. One possibility is long-range workload prediction to schedule data prefetch.

\section{REFERENCES}

[1] K. Rajamani, H. Hanson et al., "Application-aware power management," in 2006 IEEE Intl. Symp. on Workload Characterization.

[2] A. Shye, B. Scholbrock, and G. Memik, "Into the wild: studying real user activity patterns to guide power optimizations for mobile architectures," in 2009 IEEE/ACM Intl. Symp. on Microacrhitecture.

[3] G. Contreras and M. Martonosi, "Power prediction for intel xscale $\AA$ processors using performance monitoring unit events," in 2005 IEEE Intl. Symp. on Low Power Electronics and Design.

[4] K. Singh, M. Bhadauria, and S. McKee, "Real time power estimation and thread scheduling via performance counters," 2009 ACM SIGARCH.

[5] R. Zamani and A. Afsahi, "Adaptive estimation and prediction of power and performance in high performance computing," 2010 Computer Science-Research and Development.

[6] R. Grosse, R. Raina, K. H., and A. Ng, "Shift-invariance sparse coding for audio classification," 2012 arXiv preprint arXiv:1206.5241.

[7] T.-H. Lin and H.-T. Kung, "Robust and efficient representation learning with nonnegativity constraints," 2014 Intl. Conf. on Machine Learning.

[8] Binkert, Beckmann et al., "The gem5 simulator," 2011 ACM SIGARCH.

[9] A. Gutierrez, R. Dreslinski et al., "Full-System Analysis and Characterization of Interactive Smartphone Applications," in 2011 IEEE Intl. Symp. on Workload Characterization.

[10] Y. Huang, Z. Zha, M. Chen, and L. Zhang., "Moby: A mobile benchmark suite for architectural simulators," 2014 IEEE Intl. Symp. on Performance Analysis of Systems and Software (ISPASS).

[11] A. Dhodapkar and J. Smith, "Comparing program phase detection techniques," in 2006 IEEE/ACM Intl. Symp. on Microarchitecture.

[12] S. Tarsa and H.-T. Kung, "Output compression for ic fault detection using compressive sensing," in 2012 IEEE MILCOM.

[13] T.-H. Lin and H.-T. Kung, "Computing sparse representations in o (n logn) time," in 2013 Signal Proc. with Adaptive Sparse Structure Reps.

[14] R. Rubinstein, M. Zibulevsky, and M. Elad, "Eff. implement. of the K-SVD alg. using batch orthog. matching pursuit," CS Technion, 2008.

[15] J. Hartigan and M. Wong, "Algorithm as 136: A k-means clustering algorithm," 1979 Journal of the Royal Statistical Society.

[16] Y. Pati, R. Rezaiifar, and P. Krishnaprasad, "Orthogonal matching pursuit: Recursive function approximation with applications to wavelet decomposition," in 1993 Conf. on Signals, Systems and Computers.

[17] A. Septimus and R. Steinberg, "Compressive sampling hardware reconstruction," in 2010 IEEE Intl. Symp. on Circuits and Systems.

[18] D. Brooks, V. Tiwari, and M. Martonosi, Wattch: a framework for architectural-level power analysis and optimizations. 